\title{
Les Fiancés de Manzoni : radiographie d'un texte en mouvement
}

\section{Giulia Raboni}

\section{(2) OpenEdition}

1 Journals

\section{Édition électronique}

URL : https://journals.openedition.org/genesis/4645

DOI : 10.4000/genesis.4645

ISSN : 2268-1590

\section{Éditeur :}

Presses universitaires de Paris Sorbonne (PUPS), Société internationale de génétique artistique littéraire et scientifique (SIGALES)

\section{Édition imprimée}

Date de publication : 15 décembre 2019

Pagination : 101-112

ISBN : 979-10-231-0650-3

ISSN : 1167-5101

\section{Référence électronique}

Giulia Raboni, «Les Fiancés de Manzoni : radiographie d'un texte en mouvement », Genesis [En ligne], 49 | 2019, mis en ligne le 01 décembre 2020, consulté le 12 janvier 2023. URL : http:// journals.openedition.org/genesis/4645; DOI : https://doi.org/10.4000/genesis.4645 


\section{Les Fiancés de Manzoni : radiographie d'un texte en mouvement}

Giulia Raboni

L 'histoire complexe de l'élaboration des Fiancés (I promessi sposi) représente, du fait de l'importance et de la longueur de cette œuvre, le cas le plus emblématique du modus operandi d'Alessandro Manzoni. L'auteur avait préalablement adopté un même mode opératoire pour ses deux tragédies, Le Comte de Carmagnola et Adelchi, publiées entre 1820 et 1822, c'est-à-dire au début puis pendant la rédaction de ce qui constitue son unique roman. De 2006 à 2012, une équipe de travail coordonnée par Dante Isella a préparé les éditions des deux premiers manuscrits des Fiancés, accompagnées de leur dossier génétique complet, conservé presque intégralement à la Biblioteca Nazionale Braidense de Milan (seuls manquent quelques feuillets intermédiaires, perdus dès l'origine) ${ }^{1}$. L'editio princeps, accompagnée elle aussi de son dossier génétique, fait également l'objet d'une édition critique en cours de préparation. Imprimée de 1824 à 1827 par l'éditeur milanais Vincenzo Ferrario et publiée en juin 1827 - d'où le nom de Ventisettana que lui donnent les Italiens -, cette édition originale fut ensuite supplantée par la parution d'une nouvelle version du roman éditée par Redaelli entre 1840 et 1842 - surnommée, elle, la Quarantana - qui correspondait à la dernière volonté de l'auteur : la langue y avait fait l'objet d'une révision systématique, Manzoni souhaitant se conformer à l'usage du florentin parlé par la bourgeoisie de son temps ${ }^{2}$, et l'ouvrage était illustré de gravures composées par Francesco Gonin sous la supervision attentive de l'auteur.

Chacune des étapes de cette histoire éditoriale présente ainsi des spécificités propres qui requièrent un examen minutieux de la documentation génétique, particulièrement abondante du fait de l'existence des deux phases initiales manuscrites. Ces dernières proposent un défi philologique, non seulement en raison de la réélaboration profonde que subit le roman au cours de sa composition, mais aussi de l'imbrication des papiers relatifs aux deux rédactions et, plus encore, de la physionomie évolutive de la deuxième rédaction, véritable work in progress à cheval entre la première rédaction et le texte pour l'imprimeur. Il convient, pour mesurer la pertinence des perspectives nouvelles qu'ont ouvertes les études génétiques sur ce roman, de schématiser et de résumer au moins les principaux aspects de son processus d'élaboration (fig. 1).

Rappelons tout d'abord que l'ouvrage, dont l'action se situe dans la Lombardie du XVII siècle occupée par les Espagnols, conte les tribulations de deux fiancés d'origine très modeste, Renzo (Fermo dans la première version) et Lucia, dont l'union est entravée par la cruauté d'un potentat local, Don Rodrigo qui, aidé de ses braves,

1. C'est à Giuseppe Lesca que l'on doit la toute première publication complète du premier manuscrit (Gli Sposi Promessi per la prima volta pubblicati nella loro integrità di sull'autografo, Milan, Carabba, 1927). Il fallut toutefois attendre l'édition Chiari-Ghisalberti de 1954, parue dans le cadre de l'Edizione nazionale dirigée par Michele Barbi, pour disposer d'un texte critique fiable (I promessi sposi, t. III : Fermo e Lucia. Prima composizione del 1821-1823. Appendice storica su la colonna infame. Primo abbozzo del 1823, Milan, Mondadori, 1954). Aujourd'hui, le texte de référence en Italie est l'édition critique établie sous la direction de Dante Isella : I Promessi Sposi. Prima minuta (1821-1823) : Fermo e Lucia, éd. B. Colli, P. Italia et G. Raboni, Milan, Casa del Manzoni, 2006. La seule et unique édition du second manuscrit se trouve dans le volume II de l'édition critique parue en 2012 : Gli Sposi Promessi. Seconda minuta (1823-1827), éd. B. Colli et G. Raboni, Milan, Casa del Manzoni, 2012. 2. On considère les Fiancés comme l'œuvre fondatrice de la langue italienne littéraire moderne, dans la mesure où l'auteur, après un séjour en Toscane (considérée comme le berceau de l'italien) et à l'aide d'outils lexicographiques toscans tels que le Dictionnaire de l'Académie florentine de la Crusca, a épuré son style et son lexique pour donner à son roman le caractère d'archétype de la prose nationale, dans un pays marqué par la fragmentation linguistique et par la prééminence des dialectes régionaux, un pays non encore unifié politiquement, où l'italien, dont les règles écrites restaient encore mouvantes, n'était parlé que par une minorité. 


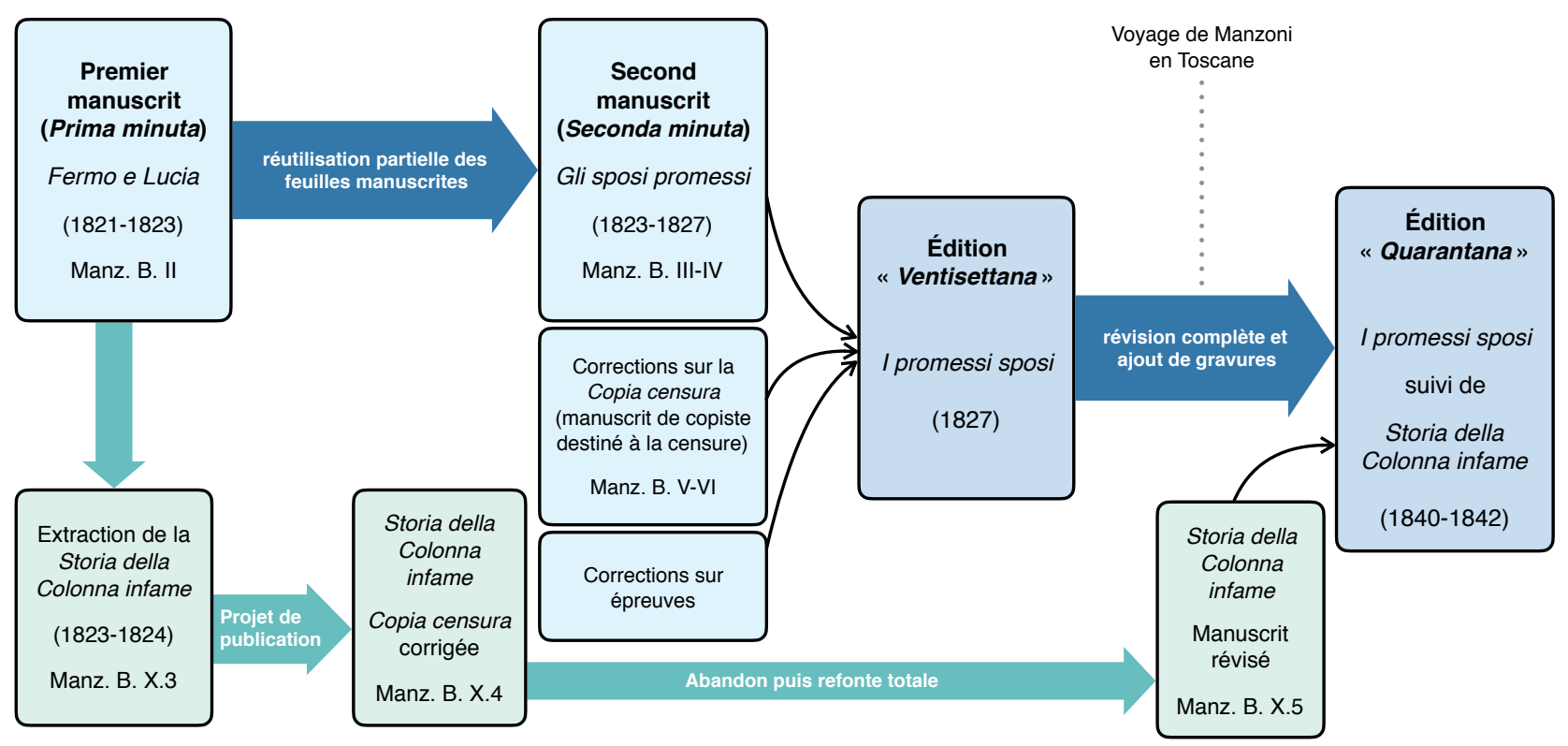

Fig. 1 : Des feuilles du premier manuscrit (Fermo e Lucia), servant de support à la seconde phase rédactionnelle du roman, sont déplacées vers le dossier contenant le second manuscrit (Gli Sposi Promessi). La première édition du roman (Ventisettana) naît de la conjonction de trois témoins : le deuxième manuscrit, la copie de copiste destinée à la censure et les premières épreuves. L'édition Quarantana marque l'aboutissement d'une nouvelle phase de révisions grammaticale, syntaxique et lexicale.

Extraite du premier manuscrit, la Storia della colonna infame est corrigée par Manzoni au moment où débute la révision du second manuscrit. L'auteur renonce ensuite à publier ce texte dans la Ventisettana et l'abandonne pendant plusieurs années. La Colonna infame, entièrement révisée, paraîtra avec la Quarantana.

tente d'enlever la jeune femme. Les deux jeunes gens sont contraints de fuir leur village et les rives du lac de Côme : le destin les sépare et ne les réunira qu'au terme de nombreuses péripéties. La rédaction du roman débute le 24 avril 1821, au beau milieu de la composition d'Adelchi, en réponse, vraisemblablement, à un moment de crise. Elle s'interrompt au terme du deuxième chapitre pendant une année environ, avant que Manzoni ne s'y attelle à nouveau jusqu'au 17 septembre 1823, date qu'il appose sur la dernière feuille de son manuscrit. Les éditeurs donnèrent à cette rédaction initiale (Biblioteca Nazionale Braidense, Manz. B. II) le titre de Fermo e Lucia, sur la foi d'une allusion tirée d'une lettre d'Ermes Visconti ${ }^{3}$; il est toutefois probable que le roman ait déjà porté, à ce stade, le titre Gli Sposi Promessi qu'il conserva jusqu'au début de l'impression.

Cette première rédaction, divisée en quatre tomes comptant au total 37 chapitres, présente une trame moins structurée et moins habilement tissée que le texte final : au terme d'une première partie consacrée aux aventures villageoises de deux protagonistes, la trame suit séparément, dans les tomes suivants, les aventures de Lucia (t. II, jusqu'au chap. 5, puis chap. 8), et de Fermo/Renzo (t. II, du chap. 6 jusqu'à l'avant-dernier, puis t. IV, chap. 4 et suivants). En conséquence, les personnages éponymes disparaissaient à tour de rôle de la scène du roman, ces éclipses étant aggravées par des digressions narratives, à l'instar des chapitres entièrement consacrés à la «Dame » de Monza ou des chapitres historiques du début du tome IV. Une telle structure, qui introduisait par endroits des contradictions dans l'articulation de l'histoire, fut pour cette raison entièrement repensée lors de la réécriture du roman. C'est pourquoi les premiers éditeurs de Fermo e Lucia, depuis Ghisalberti, décidèrent de donner à ce texte une existence autonome, qui a rendu possible une observation approfondie de ce premier moment de la composition de l'œuvre et une définition de ses spécificités intrinsèques, en relation certes avec la littérature de l'époque mais aussi avec ce que le roman manzonien allait devenir par la suite. L'étude minutieuse de la première rédaction a montré avec évidence que

3. Ermes Visconti (1784-1841), homme de lettres, élève de Vincenzo Monti, qui occupait la chaire d'éloquence à l'université de Pavie. En 1824 il succéda à Claude Fauriel dans le rôle de relecteur et d'annotateur du Premier manuscrit. Il écrivait le 3 avril 1822 au poète Gaetano Cattaneo : «Manzoni a déjà fini Adelchi depuis quelque temps, avec deux chœurs [...]. Voyons quel sera l'avis du grand traducteur. Il ne manque plus que Walter Scott traduise son roman Fermo e Lucia quand il l'aura fini», cité dans S. Casalini (éd.), E. Visconti. Dalle lettere : un profilo, "Quaderni Manzoni», Milan, Centro Nazionale di Studi Manzoniani, 2004, p. 72-73. 
le travail d'écriture de Manzoni (parfaitement documenté du reste par les dossiers génétiques de ses autres œuvres, au premier rang desquelles ses tragédies) était toujours précédé d'une intense réflexion théorique - en grande partie consignée dans sa correspondance avec le linguiste et philosophe Claude Fauriel, qui était à l'époque son ami le plus proche 4 - à laquelle la mise en œuvre concrète de son projet est empiriquement subordonnée. Ce lien étroit entre théorie et pratique s'explique tout d'abord par le fait que Manzoni inaugure en substance un genre nouveau en Italie - le roman historique - et qu'il se montre soucieux d'identifier le chemin à parcourir et les instruments à adopter afin de résorber l'écart qui s'était creusé entre une littérature italienne encore ancrée dans une culture aristocratique et élitaire, et une très large portion de la population à qui il fallait désormais offrir en partage une nouvelle grammaire morale, culturelle et linguistique, qui pût servir de carte d'identité à cette nation naissante. Il s'explique également par le fait que l'auteur élabore sa construction narrative au fil de la plume, sans s'adosser à des matériaux préexistants (plans, ébauches, notes), procédant continuellement à des réajustements entre ses principes théoriques initiaux et son œuvre telle qu'elle prend forme progressivement. Sans doute est-ce la raison pour laquelle la première rédaction avance par blocs afin d'accompagner en temps réel la naissance de l'histoire.

La réflexion théorique concerne en particulier deux thèmes centraux que Manzoni identifie immédiatement comme les données fondamentales, et tout à la fois problématiques, du nouveau genre qu'il a choisi :

- le projet d'une langue nationale et populaire,

- le rapport entre vérité et vraisemblance.

Ce second point constituait un problème théorique crucial non seulement pour la conception même du roman historique, mais aussi pour comprendre les présupposés de la pratique littéraire manzonienne. L'auteur conçoit en effet sa pratique comme un outil d'analyse des comportements humains, représentés dans un environnement historique concret (la «vérité») qui en garantit le caractère réaliste ; mais ces comportements doivent, dans le même temps, pouvoir être librement anatomisés et rester assujettis au libre arbitre, ce qui n'était évidemment pas le cas des personnages tirés de la réalité historique, comme Manzoni avait pu en faire le constat dans sa tragédie Adelchi. La mise en pratique non contradictoire de cette double exigence de vérité et de vraisemblance est rendue possible dans les Fiancés par le caractère marginal des personnages ( «Qui sait seulement qu'ils existent? Gens perdus sur la terre [...] qui ne sont à personne ${ }^{5}$ ») qui n'interfèrent pas avec le déroulement de la grande Histoire, ce qui garantit le respect scrupuleux de la vérité historique. Manzoni faisait clairement état de ce projet dans une lettre célèbre à Fauriel ${ }^{6}$ qui traduit aussi l'impréparation du narrateur, rapidement contraint d'instaurer des interactions plus étroites entre le plan de l'Histoire et celui de la fabula, et de commettre par conséquent un certain nombre d'infractions historiques plus ou moins graves (la rencontre entre des individus historiques et fictifs, la participation des personnages principaux à des événements réellement advenus), sous peine de briser l'unité de son roman. Cependant, alors même que cette œuvre est le fruit d'un projet pour ainsi dire déstabilisé en cours de route et qu'il subit d'importantes phases de réécriture tant immédiates que rétrospectives, la rédaction de Fermo e Lucia s'achève en septembre 1823 sans présenter en apparence les symptômes d'une crise, mais de simples ralentissements, dus à des approfondissements nécessaires, comme en attestent les datations du manuscrit et la correspondance de Manzoni au cours de cette période (principalement avec Fauriel, mais aussi avec Ermes Visconti, l'écrivain Tommaso Grossi, l'évêque Luigi Tosi et d'autres encore). Même le défi linguistique, auquel le romancier sait bien qu'il ne peut apporter qu'une réponse incertaine et nécessairement approximative, comme il le

\footnotetext{
4. Claude Fauriel (1772-1844) avait publié la traduction française des deux tragédies de Manzoni (Le Comte de Carmagnola et Adelghis), à Paris, en 1823.

5. A. Manzoni, Les Fiancés, trad. de l'italien par Yves Branca, Paris, Gallimard, 1995, chap. 11, p. 274.

6. Lettre en français du 3 novembre 1821 : «Pour vous indiquer brièvement mon idée principale sur les romans historiques, et vous mettre ainsi sur la voie de la rectifier, je vous dirai que je les conçois comme une représentation d'un état donné de la société par le moyen de faits et de caractères si semblables à la réalité, qu'on puisse les croire une histoire véritable qu'on viendrait de découvrir. Lorsque des événements et des personnages historiques y sont mêlés, je crois qu'il faut les représenter de la manière la plus strictement historique ; ainsi par exemple Richard Cœur de Lion me paraît défectueux dans Ivanhoé» (Alessandro Manzoni Claude Fauriel, Carteggio, éd. I. Botta, préface E. Raimondi, Milan, Centro Nazionale Studi Manzoniani, 2000, lettre 67, p. 309-310).
} 
confie à Fauriel ${ }^{7}$, trouve progressivement sa résolution en cours de rédaction : d'un côté, la richesse et la modularité de la prose manzonienne s'affinent sur un plan strictement grammatical et syntaxique, de l'autre, sur le plan lexical, l'auteur met à profit ses multiples compétences linguistiques (français, milanais, latin) pour étoffer la langue de la tradition littéraire.

Si l'on dispose, depuis qu'il existe une édition autonome de Fermo e Lucia, d'une vision globale des présupposés théoriques et des acquis poétiques progressifs de l'auteur, il a fallu attendre l'achèvement de la récente édition du second manuscrit pour que la lumière soit faite sur la phase suivante de la rédaction qui aboutit, au terme de quatre intenses années de travail, à l'édition princeps publiée en 1827 par Ferrario. Elle marque l'achèvement, à de rares exceptions près, de l'élaboration narrative du texte, qui ne fut soumis par la suite qu'à une révision linguistique et stylistique dont l'édition de 1840-1842 - la Quarantanaporte les fruits. Ce remaniement du roman, consigné pour l'essentiel sur les feuilles du second manuscrit (Biblioteca Nazionale Braidense, Manz. B. III-IV), s'étend aussi à d'autres témoins et cette dispersion complique toute tentative de reconstitution. En effet, pour mener à bien cette seconde rédaction, Manzoni utilise les brouillons de la première, qu'il déplace matériellement dans un nouveau dossier et qu'il soumet à d'importantes révisions : celles-ci sont généralement consignées dans la colonne de gauche de la page manuscrite, laissée libre à cet effet selon un usage constant chez l'auteur; mais les révisions s'intègrent par endroits à la leçon originale, par le biais de corrections intralinéaires, ce qui les rend parfois difficiles à distinguer de la leçon de base. Tel est le cas d'une large partie du tome I (fig. 2), le seul qui conserve, au fil des différentes rédactions, la même ossature narrative, et de certaines feuilles du tome III.

Par ailleurs, Manzoni, qui restait à l'évidence convaincu de la qualité générale de sa première rédaction, charge immédiatement un copiste de préparer le manuscrit à remettre à la censure autrichienne ${ }^{8}$ (Manz. B. V-VI) et lui transmet au fur et à mesure les chapitres revus, voire les simples feuilles, dans l'idée sans doute de procéder à la fin à une révision d'ensemble. Enfin, satisfait des progrès accomplis au cours de ces premiers mois de réélaboration, il lance dès le mois de juin 1824 l'impression du premier volume auprès du typographe Ferrario. Manzoni se retrouve ainsi à travailler simultanément sur trois «tableaux » :

- la rédaction du second manuscrit, qui acquiert son autonomie vis-à-vis du premier à partir du tome II, au moment où l'auteur se détache de la trame et des brouillons de Fermo e Lucia;

- la correction des parties déjà copiées pour la censure ;

- la correction des ébauches typographiques mises en page.

Cette segmentation et superposition des phases de travail explique pourquoi le second manuscrit ne constitue pas un ensemble rédactionnel autonome, un brouillon complet, comme pouvait l'être Fermo e Lucia (dont l'unique témoin a fait l'objet d'une révision suivie et rétrospective), mais se configure plutôt comme un texte en mouvement, qui accueille progressivement de nouvelles propositions et de nouvelles solutions, et dont l'harmonisation systématique est menée à bien non pas sur un unique support autographe mais sur des témoins successifs.

En raison même de la nature disparate de tels brouillons, le second manuscrit a toujours été considéré comme un répertoire de variantes ponctuelles ou de segments narratifs isolés bien plus que comme un ensemble unitaire. C'est pourquoi les premiers éditeurs du roman se sont contentés d'y puiser de quoi nourrir leurs notes et commentaires sur la Ventisettana. L'édition critique la plus récente a pris au contraire la décision d'allouer à ce manuscrit un espace éditorial autonome et de le considérer dans sa globalité, sans tenir compte de sa chronologie relative vis-à-vis des autres phases du travail rédactionnel : un tel choix contrevient

7. Ibid., p. 312 : «Pour moi, dans le désespoir de trouver une règle constante et spéciale pour bien faire ce métier, je crois cependant qu'il y a aussi pour nous une perfection approximative de style, et que pour en transporter le plus possible dans ses écrits il faut penser beaucoup à ce qu'on va dire, avoir beaucoup lu les Italiens dits classiques, et les écrivains des autres langues, les Français surtout, avoir parlé de matières importantes avec ses concitoyens, et qu'avec cela on peut acquérir une certaine promptitude à trouver dans la langue qu'on appelle bonne ce qu'elle peut fournir à nos besoins actuels, une certaine aptitude à l'étendre par l'analogie, et un certain tact pour tirer de la langue française ce qui peut être mêlé dans la nôtre sans choquer par une forte dissonance, et sans y apporter de l'obscurité. »

8. En Lombardie, alors sous domination autrichienne, une copie manuscrite des ouvrages à paraître devait obligatoirement être validée par les autorités pour que soit accordé l'admittitur préalable à l'impression. 


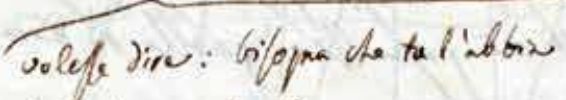
fatto ún grollo il mavrone.

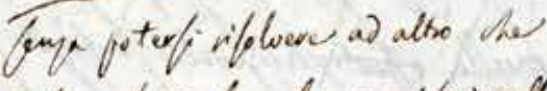

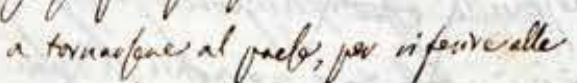
jonne, ha triftri malation

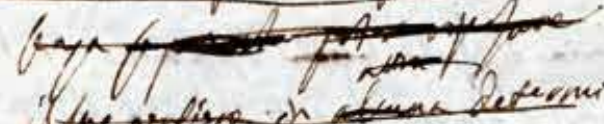

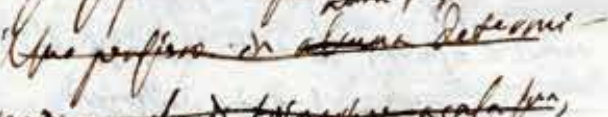

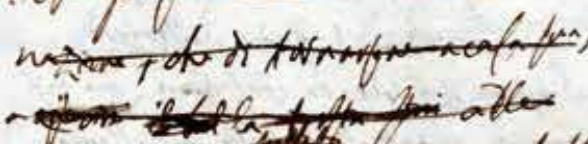

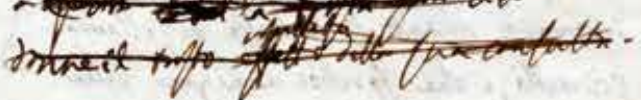

Fig. 2 : Manz. B. II, tome I, chap. 3, f. 30a. 


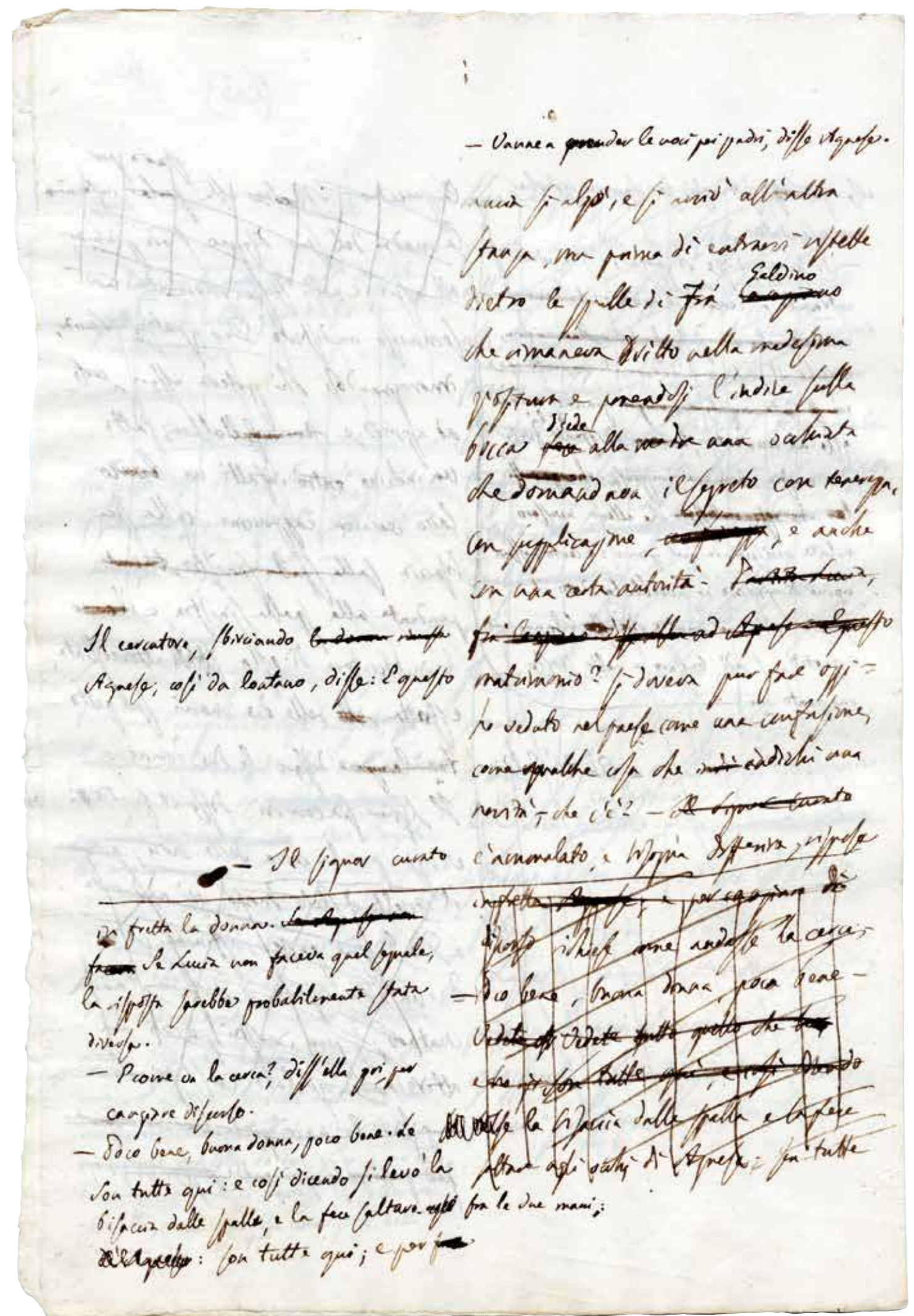

Fig. 3 et 4 (page ci-contre) : Manz. B. II, t. I, chap. 3, f. 30d,

et sa transcription dans l'édition critique de 2012 : Gli Sposi Promessi. Seconda minuta, 1823-1827, p. $46-47$.

@ ( Milan, Biblioteca nazionale Braidense. Avec l'aimable autorisation du Ministero dei beni e delle attività culturali e del turismo. Reproduction interdite 
GL1 SPOS1 PRomess!

TOMO $\mathrm{t}$ - CAPITOLO $\mathrm{HI}$

$4 \quad$ - Vanne a prender le noci pei padri, disse Agnese.

Lucia si alzò, e si avviò all'altra stanza, ma prima di entrarvi ristette dietro le spalle di Frà Galdino che rimaneva ritto nella medesima positura e ponendosi l'indice sulla bocca diede alla madre una occhiata che domandava il segreto con tenerezza, con supplicazione, e anche con una certa autorità. II cercatore, sbirciando Agnese, cosi da lontano, disse: E questo matrimonio? si doveva pur fare oggi = ho veduto nel paese come una confusione, come qualche cosa che indichi una novità; che cè̀?

- Il signor curato è ammalato, e bisogna differire, rispose in 45 fretta la donna. Se Lucia non faceva quel segnale, ha risposta sarebbe probabilmente stata diversa.

- E come va la cerca?, diss'ella poi, per cangiare discorso

- Poco bene, buona donna, poco bene. Le son tutte qui: e cosi dicendo si levò la bisaccia dalle spalle, e la fece saltare fra le due mani: son tutte qui; e per

Fig. 4

en un sens aux présupposés hiérarchiques de la philologie d'auteur et privilégie davantage la perspective, plus matérielle et synchronique, de la génétique textuelle 9 . Cette approche a en effet permis d'envisager la phénoménologie du manuscrit en accordant une plus grande attention à son statut hybride, notamment en ce qui concerne la double nature textuelle des brouillons partagés : un expédient typographique (une surbrillance grise, qui met en évidence les phrases ou les mots de la première rédaction matériellement réutilisés dans la seconde) permet de rendre immédiatement perceptibles l'écart existant entre les deux versions, mais également l'incidence du texte préexistant sur la phase de réécriture (fig. 3 et 4 ).

Cette autonomie nouvelle accordée à la deuxième rédaction a permis d'éclairer pleinement la complexité du processus de composition manzonien, en relation précisément aux principaux enjeux que l'auteur avait lucidement identifiés aux débuts mêmes de la composition. Sur le plan de la langue, tout d'abord, une comparaison directe entre le premier manuscrit et la Ventisettana ne permettait pas de dépasser le constat d'un écart très net entre le premier jet et le texte imprimé, ce qui invitait à formuler l'hypothèse d'une césure chronologique entre la fin de Fermo e Lucia et le début de la réécriture, au cours de laquelle Manzoni se serait plongé dans la lecture des classiques et du dictionnaire de l'Académie florentine de la Crusca afin de mettre au point une nouvelle recette linguistique. C'est également dans un sens trop littéral et dramatique que l'on avait lu la «Seconda introduzione scritta da ultimo », nouvelle introduction composée durant la phase intermédiaire entre l'achèvement de la première rédaction et le début de la révision, et destinée à remplacer la première, écrite en avril 1821 en même temps que les deux premiers chapitres. L'auteur y manifestait son insatisfaction vis-à-vis de la solution linguistique adoptée tout en reconnaissant ouvertement qu'il lui manquait une hypothèse de travail de substitution :

Il suffit à l'auteur que l'on juge qu'il n'a pas mal écrit par négligence envers son lecteur, par mépris du beau style épuré, qu'il n'est pas de l'espèce de ceux qui tirent quelque gloire d'écrire mal. De la gloire! À supposer que ce fût un défi difficile, ceux qui l'ont relevé sont si nombreux que chacun n'en pourrait tirer qu'une maigre gloire. J'écris mal - et que l'on pardonne à l'auteur de parler de lui : c'est le privilège des préfaces, une mince et trop juste licence accordée à la vanité de ceux qui font des livres - j'écris mal bien malgré moi; et si je connaissais la manière de bien écrire, je ne manquerais certes pas de la mettre en application. Les dons de l'esprit ne s'acquièrent pas, comme leur nom même l'indique; mais il n'est rien que je ne ferais qui soit de mon ressort pour acquérir tout ce que peuvent me procurer l'étude et la diligence ${ }^{10}$.

Or le second manuscrit montre que Manzoni se lance dans la réécriture de son roman dès l'achèvement de Fermo e Lucia et qu'il y maintient fermement ses présupposés linguistiques initiaux, avant d'opérer, à partir du chap. 5, un changement de cap décisif dont les effets s'observent directement dans la suite de cette seconde rédaction, et, pour les chapitres déjà copiés, dans les corrections apportées au manuscrit destiné à la censure. Ce changement conduit l'auteur à dépasser la recette linguistique «mixte» de sa première rédaction par le biais d'une révision pragmatique qui transpose la langue hybride du premier manuscrit, comportant des lombardismes et des gallicismes, vers le modèle toscano-littéraire de l'édition princeps au gré d'une progressive substitution des formes allotropiques, modelées en particulier sur le dialecte milanais, par des expressions toscanes qui non seulement uniformisent la physionomie linguistique du roman mais, du fait de leur vitalité expressive, stimulent l'invention de l'auteur. Prenons, à titre d'exemple, l'histoire familiale de Ludovic (entré dans les ordres sous le nom de «père Christophe», chap. 4), dont le père marchand aspire obstinément à passer pour un gentilhomme. Dans la première version, Manzoni se contente

9. En français dans le texte (note de la rédaction).

10. A. Manzoni, «Introduzione», dans I Promessi Sposi. Prima minuta (1821-1823) : Fermo e Lucia, éd. citée de 2006, p. 6 (nous traduisons). 
d'une rapide notation sociologique (probablement inspirée de Boccace ${ }^{11}$ ), certes déjà renforcée par une comparaison antiphrastique et hyperbolique entre les lubies d'un bourgeois enrichi et le drame d'un personnage shakespearien :

Sieur Ludovic (ainsi fut baptisé par son parrain celui qui prit le nom de Christophe en se faisant prêtre), sieur Ludovic était le fils d'un riche marchand de Crémone qui dans ses dernières années, veuf, sans autre enfant que ce fils, renonça au négoce, acheta des biens immobiliers, s'adonna à la vie seigneuriale, s'attacha à faire oublier qu'il avait été marchand, et il aurait voulu pouvoir l'oublier lui-même. Mais le magasin, les ballots, le grand livre et l'aune lui reparaissaient sans cesse en mémoire, comme à Macbeth l'ombre de Banco, parmi la pompe des festins et les sourires des parasites; et le malheureux passa ses dernières années dans des angoisses continuelles, persuadé à tout instant qu'on se moquait de lui, et sans jamais faire réflexion que vendre n'est pas plus honteux qu'acheter, et qu'il avait exercé cette profession ouvertement et publiquement sans le moindre remords. Il fit éduquer son fils noblement, selon les usages du temps, et autant que le lui concédaient les lois et les coutumes, et la crainte du ridicule. Il lui donna des maîtres de littérature et d'exercices cavaliers ; et il mourut, le laissant riche, et jeune homme ${ }^{12}$.

Dans la version suivante, l'auteur caractérise avec plus d'ironie encore l'obsession du personnage en insérant dans son texte la locution toscane «far orecchie da mercante» (littéralement «faire oreille de marchand», dont le sens est «faire mine de ne pas entendre ${ }^{13}$ ), qui transforme la scène en une saynète délicieusement humoristique :

[...] le magasin, les ballots, le grand livre et l'aune lui reparaissaient sans cesse en mémoire, comme à Macbeth l'ombre de Banco, et jusque parmi la pompe des festins, et les sourires des parasites. Et l'on ne saurait dire tout le soin qu'il fallait à ces malheureux pour éviter la moindre parole qui parût une allusion à l'ancien état du maître de maison. En voici un exemple : un jour, vers la fin d'un repas, au moment de la plus vive et franche allégresse, telle qu' on ne pouvait dire qui était plus heureux, de la compagnie qui s'empiffrait, ou de l'hôte qui régalait, celui-ci taquinait, d'un ton amicalement condescendant, l'un des convives, au demeurant le plus honnête mangeur du monde. Cet homme, pour ne pas être en reste, répondit sans la moindre ombre de malice, et avec une candeur vraiment enfantine : «Eh ! je fais oreille de marchand !» Il fut lui-même frappé du son de ce mot qui venait de lui échapper. Il tourna un visage timide vers le visage du maître, qui s'était rembruni. L'un et l'autre auraient voulu rattraper ce mot, mais il était trop tard. Chacun des convives pensait tout naturellement au moyen d'apaiser ce petit scandale, et de faire diversion; mais, en pensant, ils se taisaient, et le silence rendait le scandale plus manifeste; chacun voulait éviter le regard des autres; chacun sentait que tous étaient occupés de cette même pensée que tous voulaient dissimuler. La joie, ce jour-là, s'envola; et l'imprudent, ou, pour mieux dire, l'infortuné, ne fut plus jamais invité. Ce fut ainsi que le père Ludovic passa ses dernières années $[\ldots]^{14}$

La façon dont Manzoni procède ici est particulièrement significative : après un premier remaniement de l'épisode dans la colonne de gauche du manuscrit de Fermo e Lucia, il s'interrompt au moment même de transcrire l'expression toscane (fig. 5), abandonnant alors ce support, il récrit entièrement le paragraphe sur de nouvelles feuilles.

L'étude du second manuscrit a également mis au jour des éléments d'une importance capitale relatifs au rapport entre Histoire et fiction. En effet, la comparaison entre les scénarios concurrents que l'on rencontre dans les brouillons a permis de révéler l'existence, vers le milieu du roman, d'un moment de crise dans l'élaboration du projet : Manzoni, au terme d'une réécriture intégrale qui avait entrelacé les fils narratifs tout en respectant substantiellement la teneur de la première version, procède à une révision radicale qui témoigne de sa volonté d'approfondir la psychologie des personnages (la «Dame» de Monza, l'Innomé, Renzo lors de sa fuite sur l'Adda), afin de mieux rendre palpable leur évolution intellectuelle tout en accentuant leur dimension universelle. Du fait de ce changement de perspective - de l'extérieur vers l'intériorité -, la «vérité » de l'œuvre ne tient plus au respect scrupuleux de l'Histoire et de ses données documentaires, mais à la profondeur de

11. Boccace, Le Décaméron, trad. de l'italien par Giovanni Clerico, Paris, Gallimard, 2006, VII, 7, p. 599 : «Il vous faut savoir qu'il y eut jadis un gentilhomme florentin à Paris, qui s'était fait marchand par pauvreté, et auquel avait si bien réussi le commerce qu'il en était devenu très riche. De sa femme il n'avait qu'un fils, prénommé Ludovic. Et pour qu'il se tournât vers la noblesse du père et non vers le commerce, son père n'avait pas voulu le mettre dans un magasin, mais il l'avait mis avec d'autres gentilshommes au service du roi de France, où il avait appris de belles manières et de bonnes choses. »

12. Nous traduisons.

13. Manzoni avait annoté cette locution sur l'édition du dictionnaire de la Crusca qu'il possédait.

14. A. Manzoni, Les Fiancés, trad. citée, chap. 4, p. 125-126. 


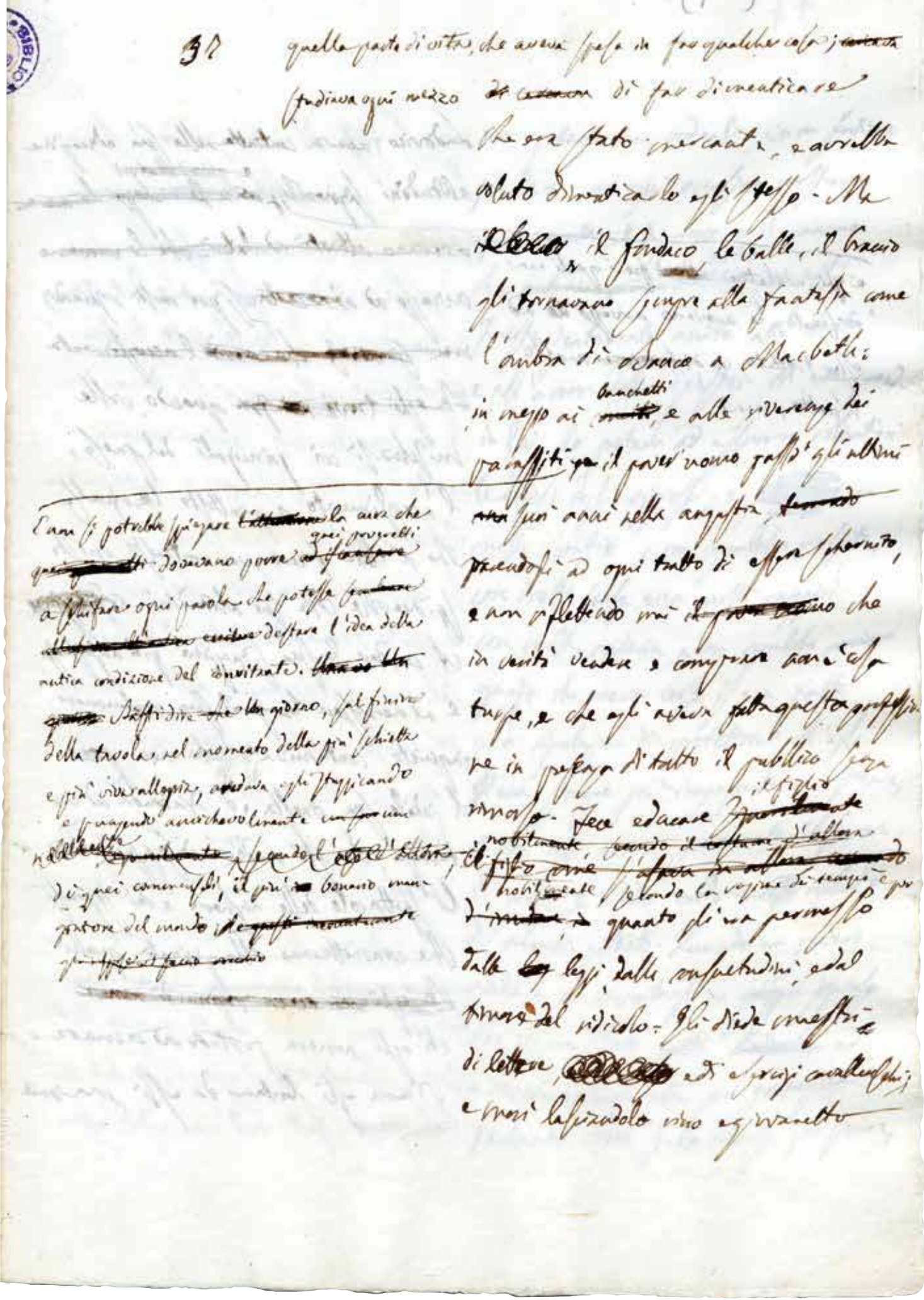

Fig. 5 : Manz. B. II, t. I, chap. 4, f. 37a, où l'on peut lire au bas de la colonne de gauche «io faccio orecchie », inachevé et rayé. (c) Milan, Biblioteca nazionale Braidense 
l'analyse des mouvements de l'âme, indépendamment des circonstances les plus contingentes. La subordination de la fiction à l'Histoire postulée initialement est inversée; le non-respect de la réalité historique est compensé par la fonctionnalisation symbolique du récit, qui déplace vers un autre plan cette quête de vérité servant de principe fondateur au roman. Cela détermine, dans la suite du texte, une plus nette accentuation du caractère allégorique du récit et, par conséquent, une plus franche démarcation entre les passages de nature proprement historique et ceux dévolus à la fiction narrative. Ainsi, dans Fermo e Lucia, un unique chapitre (IV, 2) relatait la fuite de don Abbondio, Perpetua et Agnès vers le château de l'Innomé, leur retour au village, puis les premières manifestations de la peste qui frappa le Milanais en 1630 et les investigations du Tribunal de la Salubrité. Dans le second manuscrit (puis dans la Ventisettana et la Quarantana), la matière est restructurée : le chap. 30 est entièrement consacré à l'histoire des trois personnages, tandis que les recherches de la Salubrité et le long compte rendu historique sur la progression de l'épidémie à travers le territoire lombard jusqu'à Milan sont réunis dans les chapitres suivants (3I-32).

Il paraît donc évident que l'entrelacement de l'Histoire et de la fiction, que Manzoni avait tenté tant bien que mal de mettre en œuvre dans le but de préserver l'unité de sa trame, aboutit à une profonde remise en cause de ses présupposés théoriques initiaux : l'auteur devra reconnaître que le statut du roman historique se heurte à une impossibilité même, dont il rendra compte publiquement, bien des années plus tard en 1850, dans son essai Del romanzo storico e in genere de'componimenti misti di storia e d'invenzione ${ }^{15}$; mais cette impossibilité transparaît dès l'étape de la réélaboration du roman, à la faveur de la séparation décisive qui s'y observe entre Histoire et invention, et de la revendication qui découle de la nécessaire autonomie de la création artistique. Ainsi le choix de publier le brouillon s'est-il avéré utile pour identifier avec une plus grande précision les tensions narratives et les élans contradictoires qui se sont manifestés à mesure que Manzoni rédigeait son roman; cela a permis, en outre, de comprendre la raison des hésitations et des reprises qui jalonnent la réélaboration du texte et qui, autrefois éditées de façon parcellaire dans les notes de bas de pages et dans l'apparat critique, n'offraient qu'un faible éclairage critique.
Il faut ajouter que l'étude des transformations de la trame narrative au fil de la genèse et l'analyse linguistique rendue possible par la nouvelle édition du texte montrent clairement la façon dont la Storia della colonna infame (L'Histoire de la colonne infâme) naît au sein même de Fermo e Lucia et acquiert rapidement son autonomie. Ce texte, conçu à l'origine comme une digression dans le roman, relate des événements historiques en lien avec la peste de 1630, qui sert d'arrière-plan aux chapitres 3 I-34 des Fiancés : accusés à tort d'avoir propagé l'épidémie au moyen d'un onguent jaunâtre dont ils auraient badigeonné certains murs et bancs de la ville, deux citoyens sont condamnés à mort au terme d'un procès expéditif et de tortures effroyables. C'est le fameux procès des «untori» minutieusement reconstitué par Pietro Verri dans ses Observations sur la torture (écrites en 1776-1777, publiées posthumes en 1804), dont Manzoni avait consulté les manuscrits préparatoires. À la place de la maison d'un des condamnés, rasée au sol, fut érigée à Milan une colonne à la mémoire de leur «infamie», qui ne fut abattue qu'en 1778 et qui donne son titre à l'essai historique de Manzoni.

L'Histoire de la colonne infâme, qui constituait à l'origine le chapitre 5 du tome IV de Fermo e Lucia, en est immédiatement extraite : ce texte est non seulement déjà achevé, mais copié et corrigé à nouveau en 1823 (tandis qu'une précédente hypothèse datait la révision des années 1829-1831), Manzoni envisageant de le publier conjointement à la première édition de son roman. Cette scission s'explique précisément par la crise du rapport entre Histoire et fiction dont nous avons parlé plus haut et dont on comprend qu'elle ne peut être résolue que par le choix de restituer son autonomie à la fabula, face à laquelle la Colonne infâme joue le rôle de contrepoint entièrement historique.

C'est dans l'édition de 1840 que Manzoni décide de publier la Colonne infâme à la suite de son roman, les deux textes entretenant des liens étroits : tous deux sont illustrés par les gravures de Francesco Gonin, qui renforcent la cohérence à la fois historique et sociale des personnages et des lieux, tandis qu'une seule occurrence du mot « FIN »,

15. Voir A. Manzoni, Du roman historique et, en général, des œuvres où se mêlent l'histoire et la fiction, trad. de l'italien par René Guise, dans Id., Les Fiancés, suivi de L'Histoire de la colonne infâme, et du Roman historique, Paris, Éditions du Delta, 1968, t. II, p. 279-330. 
placée au terme du second texte, vient clore l'ensemble du volume. Privé des développements narratifs que comportait la première rédaction, pensé désormais comme un essai historique dont l'objet est d'établir le champ complet des responsabilités, non seulement des juges, mais des historiens et des hommes de lettres qui perpétuèrent la funeste légende des « untori » milanais sans se soucier d'en étudier la documentation ni de dénoncer le scandale de la sentence prononcée et des tortures infligées, ce second texte vient rappeler, après le dénouement en quelque sorte heureux du roman, la brutalité de l'Histoire réelle et l'importance de la vigilance et de la responsabilité individuelles face au mal présent dans notre monde : un mal qu'aucune invention romanesque ne peut ou ne doit altérer, dont nous offrent une idée concrète et bien réelle les afflictions à l'abri desquelles aucun être, même irréprochable, ne se trouve, et que seuls la morale religieuse et le juste exercice du libre arbitre peuvent mitiger. Le célèbre «suc de toute cette histoire ${ }^{16}$ », présenté dans la conclusion du roman 17 , et la morale que formule l' auteur dans l'introduction de la Colonne infâme 18 se rejoignent et se répondent, renforçant le lien qui unit ces deux textes. Dans le même temps, les noces de l'art et de la vérité, la synthèse de leur double fonction éducative (pour les puissants) et «consolatoire» (pour les victimes), se réalisent désormais dans la perspective supérieure et transcendante de la justice divine.

\section{Traduit de l'italien par Pierre Musitelli}

16. Ibid., chap. 38 , p. 818.

17. Ibid.: «[...] ils conclurent que si les malheurs viennent certes souvent parce qu'on leur en a donné l'occasion, la conduite la plus prudente et la plus innocente ne suffit pas à les tenir éloignés; et que lorsqu'ils arrivent, par notre faute, ou sans, la confiance en Dieu les adoucit, et les rend utiles pour une vie meilleure.»

18 . « [...] lorsque, considérant plus attentivement ces faits, l'on y découvre une injustice qui aurait pu être reconnue par ceux-là mêmes qui la commettaient, une transgression des règles auxquelles eux-mêmes souscrivaient, des actes contraires aux lumières qui non seulement éclairaient leur époque, mais dont eux-mêmes, dans des circonstances similaires, firent montre par ailleurs, c'est un soulagement de penser que, s'ils ne surent pas ce qu'ils faisaient, ce fut parce qu'ils ne voulurent pas le savoir; que ce fut l'effet d'une ignorance dont l'homme se pare ou se défait selon son bon plaisir : ce qui ne constitue pas une excuse, mais une faute. Si l'on peut être involontairement la victime de faits atroces, on ne saurait en être involontairement l'auteur» (A. Manzoni, Histoire de la colonne infâme, trad. de l'italien par Christophe Mileschi, Bruxelles, Zones sensibles, 2019, «Introduction»).

GiUlia RABOnI enseigne la philologie italienne à l'université de Parme. Ses travaux portent sur la littérature des XVIe et XVIIe siècles (Teofilo Folengo, Le Tasse, Giovan Battista Marino, Gabriello Chiabrera) ainsi que sur la littérature contemporaine (en particulier sur l'œuvre de Vittorio Sereni). Elle a édité, sous la direction de Dante Isella et avec la collaboration de Paola Italia et Barbara Colli, Fermo e Lucia ainsi que le «Second manuscrit» des Fiancés. Parallèlement à ces travaux d'édition critique, Giulia Raboni a publié de nombreux essais et, plus récemment, le livre Come lavorava Manzoni (Rome, Carocci, 2017). 


\section{Les Fiancés de Manzoni : radiographie d'un texte en mouvement}

Cette étude présente les étapes de la longue gestation des Fiancés, en prêtant une attention particulière au «Second manuscrit» du roman, édité pour la première fois en 2016 et dont la situation textuelle complexe est mise en évidence : en «amont», ce manuscrit intègre matériellement certains documents de la première rédaction du roman, dont il réutilise de nombreuses pages; en «aval», il entretient un lien direct avec la révision des feuilles préparées par le copiste pour la censure, avec le début de l'impression et la correction des épreuves. Il s'agit donc d'un texte en mouvement qui implique que l'on en donne une interprétation non pas globale mais mise en regard avec les autres phases rédactionnelles du roman ; malgré cela, la publication intégrale de ce second brouillon, auparavant relégué dans les notes de l'édition princeps du roman, a permis de mieux percevoir la lente et pragmatique transformation des choix linguistiques de Manzoni jusqu'à l'édition de 1827 et de saisir les prémices de la crise du rapport entre vérité et vraisemblable qui ne sera explicitée publiquement que dans les années 1850 avec la parution de l'essai Sur le roman historique, une crise dont les indices significatifs sont la mise à l'écart puis la récriture de l'Histoire de la colonne infâme.

This study describes the stages of the gestation of The Betrothed and pays particular attention to the "Second manuscript" of the novel, published for the first time in 2016. It shows the complexity of its textual nature: on the one hand, it looks backward, comprising some of the documents belonging to the first drafting of the novel; on the other, it looks forward, being directly linked with the revision prepared by an amanuensis for censorship, and also with the beginning of the printing and the correcting of the proofs. It is therefore a mobile text, to be interpreted not globally but in relation to the other writing stages. In spite of that, however, the full publication of this second draft, that used to be confined in the notes of the princeps publication of the novel, has revealed the slow and pragmatic evolution of Manzoni's linguistic choices until the 1827 edition, as well as the first signs of the crisis in the relation between truth and verisimilitude that will only be publicly exhibited in the 1850 's with the publication of the essay About the Historical Novel. The most suggestive signs of this crisis are the putting aside and the subsequent rewriting of the History of the Column of Infamy.

Diese Studie stellt die Etappen der langen Entstehungszeit von Les Fiancés vor und widmet sich insbesondere dem 2016 erstmals herausgegebenem „Zweiten Manuskript“ des Romans, dessen komplexe Textgenese dargestellt wird. Oberflächlich betrachtet, integriert dieses Manuskript einige Dokumente aus dem Material des ersten Entwurfs des Romans, wovon zahlreiche Seiten wiederverwendet werden. Weniger sichtbar ist allerdings, dass seit Beginn des Drucks und des Korrekturlesens eine direkte Verbindung mit dem vom Kopisten für die Zensur überarbeiteten Textes bestand. Es handelt sich daher um einen Text in Bewegung, dem man mit einer globalen Interpretation nicht gerecht wird, sondern der danach verlangt, die verschiedenen redaktionellen Phasen des Romans zu berücksichtigten. Die Publikation des zweiten Entwurfs, auf den zuvor in den Fußnoten der Edition princeps verwiesen wurde, trägt dazu bei, Manzonis langsame und pragmatische Wandlung seiner Sprache bis zur Edition von 1827 besser zu erfassen. Auch die Anfänge der Krise des Verhältnisses von Wahrheit und Wahrscheinlichem, die erst in den 1850er Jahren mit der Veröffentlichung des Aufsatzes Sur le roman historique öffentlich wurde, kann nachverfolgt werden. Die charakteristischen Zeichen dieser Krise zeigen sich zuerst im Verwerfen und dann im Umschreiben der L'Histoire de la colonne infâme.
Este trabajo presenta las etapas de la larga gestación de Los novios, prestando particular atención al "Segundo manuscrito" de la novela, editado por primera vez en 2016, cuya compleja situación textual es puesta de manifiesto: hacia atrás, este manuscrito integra materialmente ciertos documentos de la primera redacción de la novela, reutilizando numerosas páginas; hacia adelante, mantiene una vinculación directa con la revisión de las páginas preparadas por el copista para la censura, con el comienzo de la impresión y las corrección de las pruebas. Se trata así de un texto en movimiento que exige una interpretación no global, sino a la luz de las otras fases redaccionales de la novela. No obstante, la publicación completa de este segundo borrador, hasta ese entonces relegado en las notas de la edición princeps de la novela, permitió percibir mejor la lenta y pragmática transformación de las elecciones lingüísticas de Manzoni hasta la edición de 1827 y de comprender las primicias de la crisis de la relación entre verdad y verosimilitud, que recién se explicitarán públicamente en los años 1850 con la publicación del ensayo Sobre la novela histórica -una crisis cuyos indicios significativos son el abandono y luego la rescritura de La historia de la columna infame.

Este estudo apresenta as etapas da longa gestação de «Os Noivos », com uma atenção particular no «Segundo manuscrito » do romance, editado pela primeira vez, em 2016, em que colocada em evidência a situação textual complexa: por um lado (em direção à sua origem, en amont), entrelaçando materialmente esse manuscrito com a primeira redação do romance, no qual ele reutilisa várias páginas; por outro, en aval, ele mantém uma ligação direta com a revisão das folhas preparadas por um copista para a censura, com o início da impressão e a correção das provas. Trata-se portanto de um texto em movimento, que implica que se faça uma interpretação não global, mas confrontada com as outras fases que compreendem a escrita do romance. Apesar disso, a publicação integral desse segundo rascunho, antes relegado às notas da edição princeps do romance, permitiu uma melhor percepção da lenta e pragmática transformação das escolhas linguísticas de Manzoni até a edição de 1827, e a compreensão das premissas da crise da relação entre verdadeiro e verossímel, que só será explícita publicamente nos anos 1850 com a publicação do ensaio "Sul romanzo storico », uma crise, cujos indícios significativos são, primeiro o distanciamento e, em seguida, a reescrita de «Colonne infâme».

I 1 saggio riassume le tappe della lunga gestazione della scrittura dei Promessi sposi con particolare attenzione al manoscritto edito per la prima volta nel 2016, della cosiddetta "Seconda minuta" rilevandone la complessa natura testuale : da un lato intrecciata materialmente all'indietro con la prima redazione del romanzo, di cui riutilizza numerosi fogli, dall'altro "in avanti" con la revisione dei fogli approntati da parte di un copista per la censura, e ancora con l'inizio della stampa e la correzione delle bozze. Malgrado lo statuto in movimento del testo, che ne richiede una fruizione non globale ma alternata alle altre fasi redazionali, la pubblicazione integrale di questo brogliaccio, finora relegato in nota alla princeps del romanzo, ha permesso di meglio percepire il lento e pragmatico mutarsi della ricetta linguistica manzoniana fino alla edizione ventisettana, e insieme di cogliere i primi germi di quella crisi del rapporto tra vero e verosimile che verrà esplicitata pubblicamente solo negli anni '50 con il saggio Sul romanzo storico, e della quale prima l'accantonamento e poi la riscrittura della Colonna infame costituiscono un indizio significativo. 